Provided for non-commercial research and education use. Not for reproduction, distribution or commercial use.

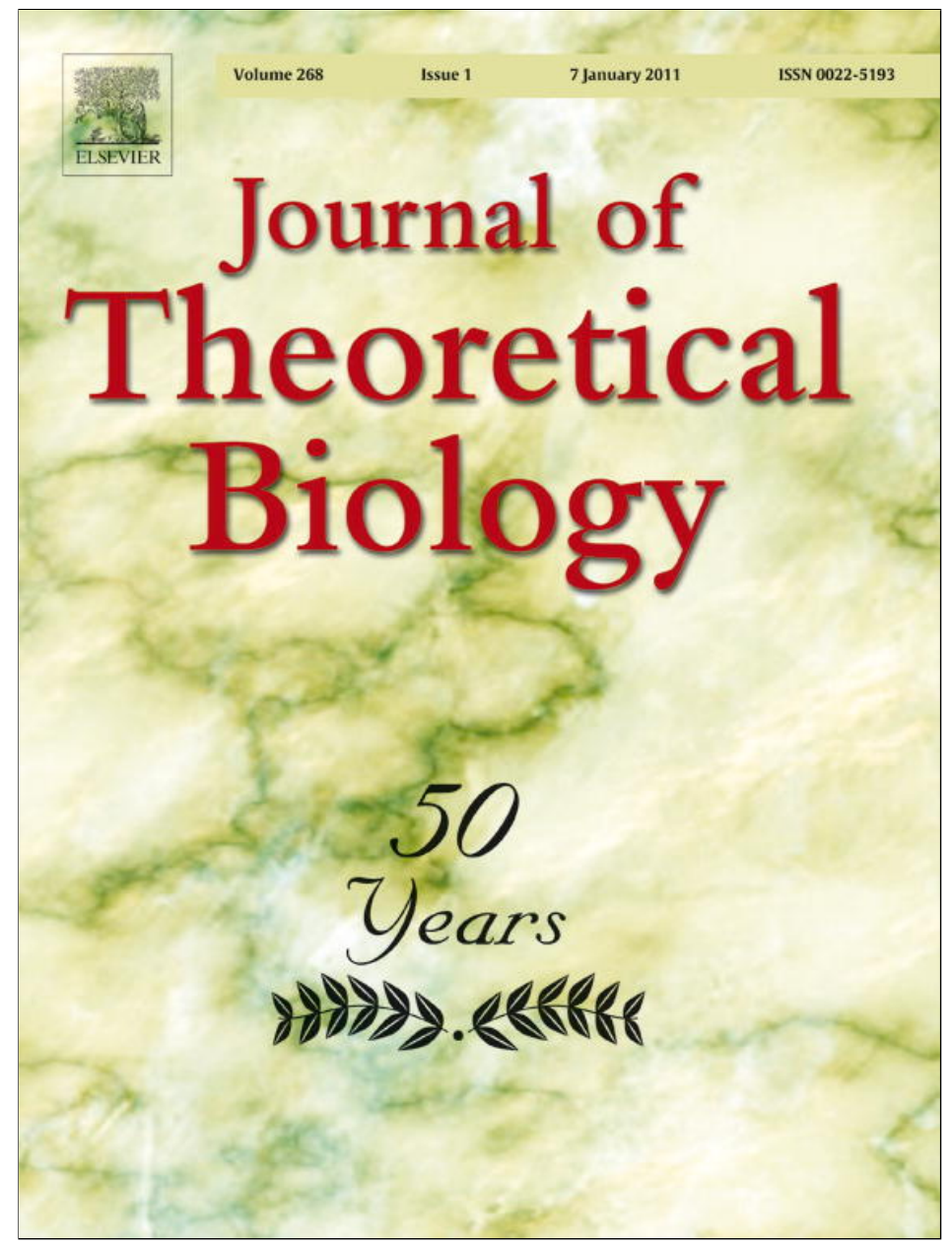

(This is a sample cover image for this issue. The actual cover is not yet available at this time.)

This article appeared in a journal published by Elsevier. The attached copy is furnished to the author for internal non-commercial research and education use, including for instruction at the authors institution and sharing with colleagues.

Other uses, including reproduction and distribution, or selling or licensing copies, or posting to personal, institutional or third party websites are prohibited.

In most cases authors are permitted to post their version of the article (e.g. in Word or Tex form) to their personal website or institutional repository. Authors requiring further information regarding Elsevier's archiving and manuscript policies are encouraged to visit: 


\title{
Modelling acidosis and the cell cycle in multicellular tumour spheroids
}

\author{
Marcus J. Tindall e,f,g,*, Louise Dyson a ${ }^{\text {a }}$ Kieran Smallbone ${ }^{\mathrm{b}, \mathrm{c}}$, Philip K. Maini ${ }^{\mathrm{a}, \mathrm{d}}$ \\ a Centre for Mathematical Biology, Mathematical Institute, 24-29 St Giles', Oxford OX1 3LB, UK \\ ${ }^{\mathrm{b}}$ Manchester Centre for Integrative Systems Biology, Manchester Interdisciplinary Biocentre, 131 Princess Street, Manchester M1 7DN, UK \\ ' School of Mathematics, University of Manchester, Oxford Road, Manchester M13 9PL, UK \\ d Oxford Centre for Integrative Systems Biology, Department of Biochemistry, South Parks Road, Oxford OX1 3QU, UK \\ e School of Biological Sciences, University of Reading, Whiteknights, Reading RG6 6BX, UK \\ ${ }^{\mathrm{f}}$ Department of Mathematics E' Statistics, University of Reading, Whiteknights, PO Box 220, Reading RG6 6AX, UK \\ ${ }^{\mathrm{g}}$ Institute for Cardiovascular and Metabolic Research, University of Reading, PO Box 218, Whiteknights, Reading RG6 6AA, UK
}

\section{A R T I C L E I N F O}

\section{Article history:}

Received 3 June 2011

Received in revised form

12 October 2011

Accepted 9 November 2011

Available online 8 December 2011

Keywords:

Acid

Mathematical model

Quiescence

Proliferating

\begin{abstract}
A B S T R A C T
A partial differential equation model is developed to understand the effect that nutrient and acidosis have on the distribution of proliferating and quiescent cells and dead cell material (necrotic and apoptotic) within a multicellular tumour spheroid. The rates of cell quiescence and necrosis depend upon the local nutrient and acid concentrations and quiescent cells are assumed to consume less nutrient and produce less acid than proliferating cells. Analysis of the differences in nutrient consumption and acid production by quiescent and proliferating cells shows low nutrient levels do not necessarily lead to increased acid concentration via anaerobic metabolism. Rather, it is the balance between proliferating and quiescent cells within the tumour which is important; decreased nutrient levels lead to more quiescent cells, which produce less acid than proliferating cells. We examine this effect via a sensitivity analysis which also includes a quantification of the effect that nutrient and acid concentrations have on the rates of cell quiescence and necrosis.
\end{abstract}

Crown Copyright (c) 2011 Published by Elsevier Ltd. All rights reserved.

\section{Introduction}

Multicellular tumour spheroids (MCTS) are three-dimensional cellular aggregates which mimic many of the characteristics of in vivo avascular tumours (Mueller-Klieser, 1997). They have been the focus of research for experimentalists and applied mathematicians over the past 30 years (Araujo and McElwain, 2004; Roose et al., 2007). Whilst able to mimic many characteristics of in vivo tumours, for instance the observed spatial variation in oxygen and glucose concentrations from the outer to inner regions (KunzSchugart et al., 1998; Mueller-Klieser, 1997), MCTS are not widely used in cancer drug discovery, due to the cell culture techniques being more complex than standard 2D monolayers (Tung et al., 2011).

Initial mathematical modelling work in the area focused on simple models describing MCTS growth in the context of nutrient delivery to the tumour (Burton, 1996; Greenspan, 1972). With time and further experimental understanding a number of continuum mathematical models have been developed which have focused on the effect certain biological mechanisms (biochemical

\footnotetext{
* Corresponding author at: Department of Mathematics \& Statistics, University of Reading, Whiteknights, PO Box 220, Reading, RG6 6AX, UK.

Tel.: +440118 378 8992; fax: +4401189313423.

E-mail address: m.tindall@reading.ac.uk (M.J. Tindall).
}

and biomechanical) have on MCTS development (see, for example Breward et al., 2002; Netti et al., 1995; Ward and King, 1997). The work presented here involves mathematical modelling of two different aspects of tumour growth; the cell cycle and acidosis and the effects both of these have on MCTS growth.

The cell cycle is a series of tightly regulated biochemical events which control the growth and development of a cell. To summarise: cells grow during $G_{1}$ phase before entering a period during which their DNA is synthesised (S-phase). $G_{2}$, a short period following S-phase, allows the cell time to prepare for cell division, involving splitting of the DNA spindle and physical division of the cell in two (M-phase). The newly generated cells may enter a period of extended time without further proliferation. Such cells are defined to have entered the quiescent G0 phase. In tumour biology entering such a phase is usually driven by factors external to the cell, for instance a decrease in growth factors or nutrient deprivation. Cells may undergo two basic forms of cell death; apoptosis or necrosis. Apoptosis is a decision by a cell to commit cell 'suicide'. In doing so the cell shrinks to form an apoptotic body which is removed by the immune system. Physiological events, such as decreased nutrient concentration within the tumour or high acidity, can have harmful effects on quiescent cells and may eventually lead to necrosis; the breaking down of the cellular wall and release of cell contents into the extracellular environment. 
Recent experimental and mathematical modelling work has elucidated the importance of $\mathrm{pH}$ levels on tumour morphology. Noninvasive magnetic resonance (MR) techniques have been developed to measure both intracellular $\mathrm{pH}\left(\mathrm{pH}_{I}\right)$ and extracellular $\mathrm{pH}\left(\mathrm{pH}_{X}\right)$ of human and animal tissues (Gillies et al., 2004, 2002). Virtually all tumour $\mathrm{pH}$ data to date show an acidic $\mathrm{pH}_{X}$ and alkaline $\mathrm{pH}_{I}$ relative to normal tissue. Moreover, it is found that the $\mathrm{pH}_{X}$ becomes more acidic as the tumour grows, consistent with reduced perfusion (Gillies et al., 2002). Clinical specimens have shown that these changes have a molecular basis in upregulation of glucose transporter 1 and the $\mathrm{Na}^{+} / \mathrm{H}^{+}$exchanger (Gatenby et al., 2007); the developed mathematical models (Smallbone et al., 2007) have shown excellent agreement with experimental data on the distribution of upregulated cells.

There exists a growing number of mathematical models in the tumour literature which have been developed to understand the role of the cell cycle in tumour growth. These include dynamic models which encorporate simple (Bajzer et al., 1997; Bertuzzi et al., 1981; Cojocaru and Agur, 1992; Hillen et al., 2010) or complex (Zhao and Ricci, 2010) descriptions of the cell cycle, the spatiotemporal distribution of the cell cycle state within MCTS (Billy et al., 2009; Jeon et al., 2010; Mahmood et al., 2011; Tindall and Please, 2007; Tindall et al., 2008) and therapeutic interventions (Billy et al., 2009; Zhao and Ricci, 2010). To our knowledge no mathematical model currently exists which has considered how nutrient and acidosis levels affect the spatiotemporal cell cycle state of tumour cells and dead cell material within an avascular tumour.

In this paper we consider a mathematical model of a MCTS which includes a simple model of the cell cycle, where cells are considered to exist in either a proliferating, quiescent or dead cell (due to necrosis or apoptosis) state. The model includes a description of the nutrient and acid concentration within the MCTS. In what follows, the effect these have on the distribution of proliferating cells, quiescent cells and dead cell material and the overall tumour size, is investigated.

\section{Model formulation}

Let $P(\mathbf{x}, t), Q(\mathbf{x}, t)$ and $M(\mathbf{x}, t)$ represent the density of proliferating cells, quiescent cells and dead cell material per unit volume, respectively, whose mass conservation is described by

$P_{t}+\nabla \cdot(\mathbf{u} P)=\left(K_{B}(C)-K_{Q}(C, H)-K_{A}\right) P+K_{P}(C) Q$,

$Q_{t}+\nabla \cdot(\mathbf{u} Q)=K_{Q}(C, H) P-\left(K_{D}(C, H)+K_{P}(C)\right) Q$,

$M_{t}+\nabla \cdot(\mathbf{u} M)=K_{A} P+K_{D}(C, H) Q-\lambda M$,

where $\mathbf{u}(\mathbf{x}, t)$ represents the local velocity of the cells and $K_{I}$ $(I=B, P, Q, D, A)$ are cell cycle transition, or birth/death rates, which we assume are dependent upon the local diffusible nutrient $C(\mathbf{x}, t)$ and extracellular hydrogen ion (acid) concentration $H(\mathbf{x}, t)$. We assume that dead cell material is lost from the tumour at a constant rate $\lambda$ (as first observed in Greenspan, 1972).

We note that our work differs from other models by explicitly accounting for proliferating cells, quiescent cells and dead cell material. Comparative papers (Ward and King, 1997, 1998) only account for live and dead cells within an MCTS. Although we have recently considered the effect of different spatial velocities, dependent upon the cell cycle state of the cell and the cell's local extracellular nutrient gradient (Tindall et al., 2008), we have here, for simplicity, assumed that all cells move with the same spatial velocity. This assumption reduces the complexity of having to account for varying cell cycle state structures, as a result of the varying chemotactic response to the local environment within the tumour.

We will take simple expressions for the $K_{I}$ which capture the qualitative behaviour:

$K_{B}(C)=\bar{k}_{B} C$,

$K_{P}(C)=\bar{k}_{P} C$,

$K_{Q}(C, H)=\bar{k}_{Q}\left(C_{\infty}-C\right)+\bar{k}_{Q}^{\prime}\left(H-H_{\infty}\right)$,

$K_{D}(C, H)=\bar{k}_{D}\left(C_{\infty}-C\right)+\bar{k}_{D}^{\prime}\left(H-H_{\infty}\right)$,

$K_{A}=\bar{k}_{A} C_{\infty}$

Here $K_{B}(C)$ represents the rate of cell birth, $K_{P}(C)$ is the rate of cell transfer from the quiescent to proliferating compartments, $K_{Q}(C, H)$ is the rate at which cells move from the proliferating to quiescent compartment (quiescence), $K_{D}(C, H)$ is the rate of cell death from the quiescent cell compartment (necrosis), $K_{A}(C)$ is cell death from the proliferating cell compartment (apoptosis) and $C_{\infty}$ and $H_{\infty}$ denote the concentration, respectively, of nutrient and acid at the tumour boundary which are assumed to be constant.

Acidification leads to death of normal cells due to activation of p53-dependent apoptosis pathways, as well as loss of function of critical pH-sensitive genes (Park et al., 1999; Williams et al., 1999). Tumour cells, however, may be relatively resistant to acidic $\mathrm{pH}_{\mathrm{X}}$. Whilst normal cells die in environments with a persistent $\mathrm{pH}$ below about 7 , tumour cells continue to proliferate in a relatively acidic medium ( $\mathrm{pH}$ 6.8) (Casciari et al., 1992). Beyond this point quiescence and eventually necrosis occur (Patel et al., 2001). This biological knowledge is reflected in the monotonic increase of quiescence $K_{Q}$ and necrosis $K_{D}$ with $H$.

Given that the rate of diffusion of nutrient throughout the spheroid is rapid compared to the time scale of growth, we adopt the standard quasi steady-state assumption (Ward and King, 1997):

$D_{C} \nabla^{2} C=\sigma_{C}\left(P+\epsilon_{C} Q\right) C$.

This equation has two nutrient consumption terms, one relating to proliferating cells $\left(\sigma_{C}\right)$ and the other to quiescent cells $\left(\sigma_{C} \epsilon_{C}\right)$. Here $D_{C}$ is the nutrient diffusion coefficient.

In the case of acid diffusion throughout the spheroid, we also make a quasi steady-state assumption:

$D_{H} \nabla^{2} H=-\left(P+\epsilon_{H} Q\right)\left(\sigma_{H}+\sigma_{H}^{\prime}\left(C_{\infty}-C\right)\right)$,

where the acid diffuses at a rate $D_{H}$ and $\sigma_{H}$ and $\sigma_{H} \epsilon_{H}$ represent the production of acid by proliferating and quiescent cells, respectively. Note that $C \leq C_{\infty}$ and $H \geq H_{\infty}$, given the respective boundary conditions and application of the maximum principle.

In Eqs. (9) and (10), $\epsilon_{C} \ll 1$ and $\epsilon_{H} \ll 1$, representing the fact that quiescent tissue is essentially metabolically inactive, consuming significantly less oxygen than its proliferating counterpart and producing significantly fewer hydrogen ions. Tumours rely on anaerobic metabolism and hence produce acid at a rate $\sigma_{H}$ under normoxic conditions (the Warburg (1930) effect); nonetheless, as oxygen levels decrease, acid production increases linearly at rate $\sigma_{H}^{\prime}$ (the Pasteur effect, Racker, 1974). Whilst more complex descriptions of tumour metabolism are possible (see, for example Bertuzzi et al., 2007; Forbes et al., 2006), in this form the size of the parameter space remains tractable.

We adopt the common assumption that the tumour is spherical and thus we will consider solutions in the one-dimensional spherical polar coordinates regime (see Section 4). This assumption allows us to determine the motion of the cells by noting that 
there is no additional space in the tumour (incompressibility) so that

$N=P+Q+M$,

where $N$ is a constant. This also assumes that cells have similar size. A mass balance equation can then be found by adding Eqs. (1), (2) and (3) and then using (11) to obtain

$\nabla \cdot \mathbf{u}=\frac{1}{N}\left(K_{B}(C) P-\lambda M\right)$.

Throughout this model derivation, we have assumed that various processes follow simple, linear dynamics. It can be argued that these assumptions are too unrealistic to represent complex biological phenomena such as these. However, these processes are poorly understood and, as a first approximation, an assumption of linearity is sufficient to capture qualitatively similar monotonic behaviour (e.g. cell death decreasing with oxygenation and increasing with acidity in Eq. (7)). We would not expect these assumptions to have a marked effect on the model's conclusions.

\subsection{Non-dimensionalisation}

Eqs. (1), (2), (9)-(12) are non-dimensionalised according to $\xi=r / L, \quad \tau=t / T, \quad p=P / N, \quad q=Q / N$,

$c=C / C_{\infty}, \quad h=\left(H-H_{\infty}\right) / H_{0} \quad$ and $\quad v=T u / L$,

where $L=\sqrt{D_{C} /\left(N \sigma_{C}\right)}$ and $T=1 /\left(k_{B} C_{\infty}\right)$ represent length and time scales, respectively. Due to our problem being spherically symmetric we introduce the radial co-ordinate $r$. The acid concentration is scaled with respect to $H_{0}=\left(\sigma_{H} D_{C}\right) /\left(\sigma_{C} D_{H}\right)$. Our dimensional system of equations in spherical polar coordinates in the one-dimensional radial direction become

$\frac{\partial p}{\partial \tau}+\frac{1}{\xi^{2}} \frac{\partial}{\partial \xi}\left(\xi^{2} v p\right)=\left(\kappa_{b}(c)-\kappa_{q}(c, h)-\kappa_{a}\right) p+\kappa_{p}(c) q$,

$\frac{\partial q}{\partial \tau}+\frac{1}{\xi^{2}} \frac{\partial}{\partial \xi}\left(\xi^{2} v q\right)=\kappa_{q}(c, h) p-\left(\kappa_{d}(c, h)+\kappa_{p}(c)\right) q$,

$m=1-p-q$,

$\frac{1}{\xi^{2}} \frac{\partial}{\partial \xi}\left(\xi^{2} \frac{\partial c}{\partial \xi}\right)=\left(p+\epsilon_{C} q\right) c$

$\frac{1}{\xi^{2}} \frac{\partial}{\partial \xi}\left(\xi^{2} \frac{\partial h}{\partial \xi}\right)=-\left(p+\epsilon_{H} q\right)\left(1+\sigma_{h}(1-c)\right)$,

$\frac{1}{\xi^{2}} \frac{\partial}{\partial \xi}\left(\xi^{2} v\right)=\kappa_{b}(c) p-\lambda^{\prime} m$, where the non-dimensional rates are given by

$\kappa_{b}(c)=c, \quad \kappa_{p}(c)=k_{p} c, \quad \kappa_{q}(c, h)=k_{q}(1-c)+k_{q}^{\prime} h$,

$\kappa_{d}(c, h)=k_{d}(1-c)+k_{d}^{\prime} h, \quad \kappa_{a}=k_{a} \quad$ and $\quad \lambda^{\prime}=\frac{\lambda}{k_{B} C_{\infty}}$,

and the remaining non-dimensional parameters are

$k_{i}=\frac{\bar{k}_{I}}{\bar{k}_{B}}, \quad \sigma_{h}=\frac{\sigma_{H}^{\prime} C_{\infty}}{\sigma_{H}}, \quad k_{d}^{\prime}=\frac{\bar{k}_{D}^{\prime} H_{0}}{\bar{k}_{B} C_{\infty}} \quad$ and $\quad k_{q}^{\prime}=\frac{\bar{k}_{q}^{\prime} H_{0}}{\bar{k}_{B} C_{\infty}}$

for $i=p, q, d, a$.

We impose the following boundary and initial conditions. For boundary conditions on $C$ and $H$ we let $C(\mathbf{x}, t)=C_{\infty}$ and $H(\mathbf{x}, t)=H_{\infty}$. These assume the spheroid grows in a nutrient rich medium whose metabolite concentration remains constant. For the initial conditions we assume all cells are initially proliferating, i.e. $P=N, Q=0, M=0$. For simplicity we assume no cell shedding.

On non-dimensionalising the initial and boundary conditions become

$c_{\xi}(0, \tau)=h_{\xi}(0, \tau)=0, \quad c(\Gamma(\tau), \tau)=1, \quad h(\Gamma(\tau), \tau)=0$,

$v(0, \tau)=0, \quad p(\xi, 0)=c(\xi, 0)=1, \quad q(\xi, 0)=h(\xi, 0)=0$,

where $\Gamma(\tau)=R(t / T) / L$ is the non-dimensional spheroid radius with

$v(\Gamma, \tau)=\frac{d \Gamma}{d \tau} \quad$ and $\quad \Gamma(0)=\Gamma_{0}$,

where $R(t)$ is the dimensional radius of the tumour.

\subsection{Parameter estimation}

The model is dependent on eleven non-dimensional parameters as set out in Table 2. These may be estimated from parameters available in the literature as shown in Table 1.

Values for the cell cycle rates specified in the model are not readily available and as such we have determined these as follows. By definition, each time unit $\tau$ corresponds to one full cell cycle period, which we take to be twelve hours. From this and $C_{\infty}$ we can determine $\bar{k}_{B}=4.6 \times 10^{-4} \mathrm{mM}^{-1} \mathrm{~s}^{-1}$ which is in good agreement with experimentally quoted values (Kumei et al., 1989).

Previous authors have taken $k_{p}=0.05$ and $k_{q}=0.9$ (Tindall and Please, 2007), which assumes a high rate of quiescence and few cells returning from the quiescent to proliferating compartment. Using this value of $k_{q}$, and assuming that $\kappa_{q}\left(c_{Q}, 0\right)=\kappa_{q}\left(1, h_{Q}\right)$, allows us to calculate $k_{q}^{\prime}=4 \times 10^{-2}$. Here $c_{Q}$ and $h_{Q}$ are the respective nutrient and acid concentrations at which cells become

Table 1

Dimensional parameter values.

\begin{tabular}{|c|c|c|c|}
\hline Parameter & Value & Description & Reference \\
\hline$D_{C}$ & $1.5 \times 10^{-5} \mathrm{~cm}^{2} \mathrm{~s}^{-1}$ & Nutrient (oxygen) diffusion coefficient & Nichols and Foster (1994) \\
\hline$\sigma_{C} N C_{\infty}$ & $2.2 \times 10^{-1} \mathrm{mM} \mathrm{s}^{-1}$ & Tissue nutrient (oxygen) consumption rate & Banaji et al. (2005) \\
\hline$\epsilon_{c}$ & 0.1 & Quiescent:proliferating nutrient ratio & Bredel-Geissler et al. (1992) \\
\hline$\epsilon_{H}$ & 0.01 & Quiescent:proliferating metabolic ratio & Patel et al. (2001) \\
\hline$C_{\infty}$ & $5 \times 10^{-2} \mathrm{mM}$ & Normal nutrient (oxygen) concentration & Banaji et al. (2005) \\
\hline$D_{H}$ & $1.1 \times 10^{-5} \mathrm{~cm}^{2} \mathrm{~s}^{-1}$ & Hydrogen ion diffusion coefficient & Patel et al. (2001) \\
\hline$N \sigma_{H}$ & $5 \times 10^{-5} \mathrm{mM} \mathrm{s}^{-1}$ & Hydrogen ion production rate & Patel et al. (2001) \\
\hline$H_{\infty}$ & $5.6 \times 10^{-5} \mathrm{mM}$ & Normal hydrogen ion concentration & Patel et al. (2001) \\
\hline$R_{0}$ & $5-50 \mu \mathrm{m}$ & Tumour cell radius & Anderson (2005) \\
\hline$c_{Q}$ & $10^{-1}$ & Non-dimensional nutrient-induced quiescence threshold & Höckel and Vaupel (2001) \\
\hline$c_{D}$ & $5 \times 10^{-2}$ & Non-dimensional nutrient-induced necrosis threshold & Anderson (2005) \\
\hline$H_{Q}$ & $4 \times 10^{-4} \mathrm{mM}$ & Acid-induced quiescence threshold & Patel et al. (2001) \\
\hline$H_{D}$ & $10^{-3} \mathrm{mM}$ & Acid-induced necrosis threshold & Patel et al. (2001) \\
\hline$\sigma_{0: 1}$ & 1.6 & Anoxic:normoxic acid production ratio & Schornack and Gillies (2003) \\
\hline$\lambda$ & $0.05 /$ day & Rate of removal of dead cell material & Tanaka et al. (2009) \\
\hline
\end{tabular}


quiescent as given in Table 1 . This assumes that the rate of quiescence at the quiescent threshold is the same, whether due to nutrient deprivation or acid concentration. In order to calculate the non-dimensional parameters $h_{Q}$ and $h_{D}$, we have used Eq. (21) to obtain $H_{0}=1.5 \times 10^{-5} \mathrm{mM}$, and then using Eq. (13), $h_{Q}=20$ and $h_{D}=60$ follow.

To calculate $k_{d}$ and $k_{d}^{\prime}$ we use the necrosis thresholds, $c_{D}$ and $h_{D}$ (see Table 1) and assume the point at which cells die and resume proliferation are equivalent, i.e. $\kappa_{d}=\kappa_{p}$. This leads to $k_{d}=5 \times$ $10^{-2}$ and $k_{d}^{\prime}=8 \times 10^{-4}$. We have chosen a higher death rate of $k_{d}=0.2$ in order to demonstrate particular features of the model as discussed in Section 3. Investigations have shown that widespread apoptosis occurs in cells fixed mid-cycle after around one week, or 14 time units (Meikrantz and Schlegel, 1995). As such, we approximate $k_{a}=1 / 14=7 \times 10^{-2}$.

For the rate of dead cell matter lost from the tumour, we have consulted the work of Tanaka et al. (2009) who used a value of $\lambda=0.05 /$ day in modelling glioma tumours. This leads to a nondimensional value of $\lambda^{\prime}=0.6$. We have chosen a slightly lower value $\left(\lambda^{\prime}=0.1\right)$ to demonstrate specific features of the model, in particular the distribution of quiescence cells at the centre of the tumour, as discussed in the next section.

From Eq. (18) the anoxic $(c=0)$ to normoxic $(c=1)$ acid production ratio is $1+\sigma_{h}$. Thus from Table $1, \sigma_{h}=\sigma_{0: 1}-1=0.6$.

\section{Model analysis}

Before turning to numerical solutions of our model we consider how the boundary of the tumour behaves given the interplay between the three cell cycle state compartments-proliferating, quiescent and dead cell matter. We know from the boundary conditions that $c=1$ and $h=0$. Thus Eqs. (1)-(3) reduce to

$p_{\tau}=p\left(1-p-k_{a}\right)+k_{p} q+\lambda^{\prime} p m$,

$q_{\tau}=-p q+\lambda^{\prime} q m-k_{p} q$,

$m_{\tau}=-m p+\lambda^{\prime} m^{2}+k_{a} p-\lambda^{\prime} m$

on the boundary of the tumour, where the Eulerian flux terms are zero. The steady-states of this system of equations are given by

$\left(p_{1}^{*}, q_{1}^{*}, m_{1}^{*}\right)=(0,0,1), \quad\left(p_{2}^{*}, q_{2}^{*}, m_{2}^{*}\right)=\left(1-\frac{k_{a}}{1+\lambda^{\prime}}, 0, \frac{k_{a}}{1+\lambda^{\prime}}\right)$

$\left(p_{3}^{*}, q_{3}^{*}, m_{3}^{*}\right)=\left(\frac{k_{p}\left(\lambda^{\prime}-k_{p}\right)}{k_{p}-\left(1-k_{a}\right) \lambda^{\prime}} \frac{1}{k_{p}}\left(p_{3}^{*}\left(p_{3}^{*}+k_{a}-1\right)-\lambda^{\prime} p_{3}^{*} m_{3}^{*}\right), \frac{k_{a} k_{p}}{k_{p}-\left(1-k_{a}\right) \lambda^{\prime}}\right)$.

Both $\left(p_{1}^{*}, q_{1}^{*}, m_{1}^{*}\right)$ and $\left(p_{3}^{*}, q_{3}^{*}, m_{3}^{*}\right)$ are biologically unrealistic. In the first case all of the tumour dies and in the second case we must have $k_{p}>\left(1-k_{a}\right) \lambda^{\prime}$-well outside our parameter range (see Table 2). We thus ignore these and consider the more realistic case of $\left(p_{2}^{*}, q_{2}^{*}, m_{2}^{*}\right)$ which shows the tumour boundary density varies dependent upon the rate of apoptosis and the rate at which dead cell material is removed from the tumour. If these are negligible then the boundary condition of previous authors, e.g. Ward and King (1997), holds, but in the next section we consider cases where $k_{d}$ and $\lambda^{\prime}$ are large enough to affect the densities of live and dead cells at the surface as the tumour grows.

\section{Model simulations and results}

Numerical solutions to the system of Eqs. (14)-(19), with the respective boundary and initial conditions, were found by applying the method of characteristics to Eqs. (14) and (15) and the Successive Over Relaxation (SOR) method to Eqs. (17) and (18).
Table 2

Non-dimensional model parameters.

\begin{tabular}{ll}
\hline Parameter & Value \\
\hline$\Gamma_{0}$ & 3 \\
$\epsilon_{C}$ & 0.1 \\
$\epsilon_{H}$ & $10^{-2}$ \\
$\sigma_{h}$ & 0.6 \\
$k_{p}$ & $5 \times 10^{-2}$ \\
$\lambda^{\prime}$ & 0.1 \\
$k_{q}$ & 0.9 \\
$k_{q}^{\prime}$ & $4 \times 10^{-2}$ \\
$k_{d}$ & 0.2 \\
$k_{d}^{\prime}$ & $8 \times 10^{-4}$ \\
$k_{a}$ & $7 \times 10^{-2}$ \\
\hline
\end{tabular}

The finite volume method was used to solve Eq. (19). Before applying each of these methods, the governing equations were rescaled to a fixed grid by the transformation $\eta=\xi / \Gamma(\tau)$ given the moving boundary nature of the problem. The parameters used are those shown in Table 2. Model solutions are plotted at $t=1,5,10$, 40 and 50 days, respectively. Although we solve the dimensionless system (14)-(19), for illustrative purposes our results are presented in dimensional form.

Typical model results are shown in Fig. 1(a)-(f). As the spheroid grows, it develops the three distinct regions of proliferating cells on the exterior (Fig. 1(a)), quiescent cells towards the inner regions (Fig. 1(b)) and a mainly dead core of necrotic and apoptotic material (Fig. 1((c)). We note that dead cell material is found throughout the three regions due to the assumed constant rate of apoptosis. The spatial velocity profile of the cells is shown in Fig. 1(d) and is in agreement with results found elsewhere, e.g. Ward and King (1997). We note our numerical solutions concur with the boundary values given by $\left(p_{2}^{*}, q_{2}^{*}, m_{2}^{*}\right)$ in Eq. (27).

A comparison of the model with and without acid present is shown in Fig. 2. In each case the proliferating, quiescent and dead cell distributions, cell velocity, nutrient and acid concentration profiles were qualitatively equivalent to Fig. 1(a)-(f). Including acid in the tumour had no impact on the tumour size or the radial velocity profile. This is because the rates of necrosis and quiescence by acidosis are considerably smaller (three orders of magnitude) than the rates due to a decrease in nutrient concentration.

We also investigated varying the rate of necrosis $k_{d}$ and the rate at which dead cell matter $\lambda^{\prime}$ is lost from the tumour. A necrosis rate lower than that used here, i.e. $0.05 \leq k_{d} \leq 0.2$ led to more quiescent cells accumulating at the centre of the tumour and an increase in tumour size. Likewise an increase led to a smaller tumour and an even thinner quiescent region than shown in Fig. 1(b). Increasing $\lambda^{\prime}$ means dead matter is lost faster from the tumour, leading to a smaller tumour with more quiescent cells at the centre and less necrotic material than that shown in Fig. 1(b) and (c). These results show that the balance between these two rates has an important affect on the distribution of quiescent cells within the tumour. Our final parameter set was chosen to demonstrate the general MCTS cell cycle structure that of a mainly necrotic core with a small proliferating rim and intervening quiescent region. Variations in these parameters, and thus the quiescent cell and dead cell matter distribution, did not have an effect on drawing conclusions from the results which follow.

\subsection{Nutrient consumption and acid production}

We wish to understand the effect that different rates of nutrient consumption and acid production by proliferating and quiescent cells, respectively, have on the overall growth and development of the tumour. In Fig. 3, we vary the value of $\epsilon_{C}$, 
a

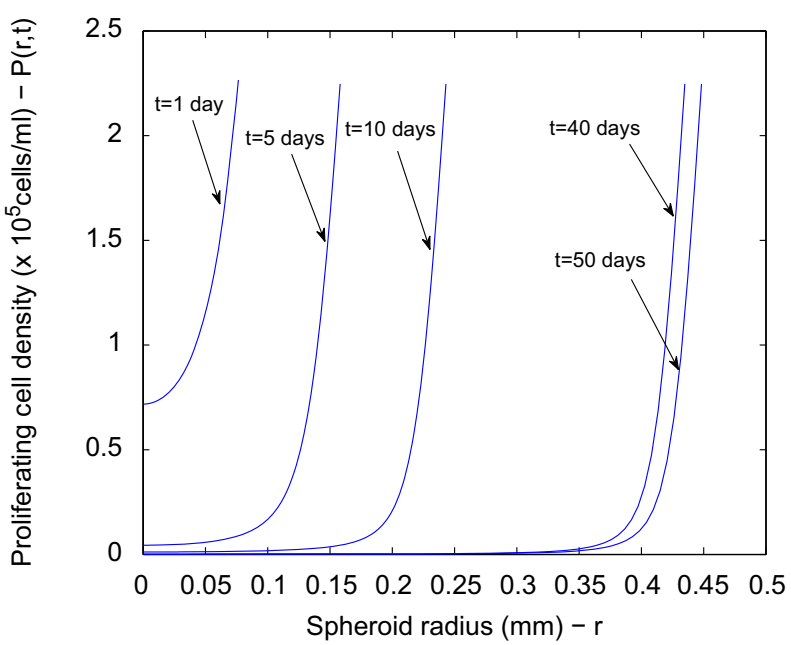

c

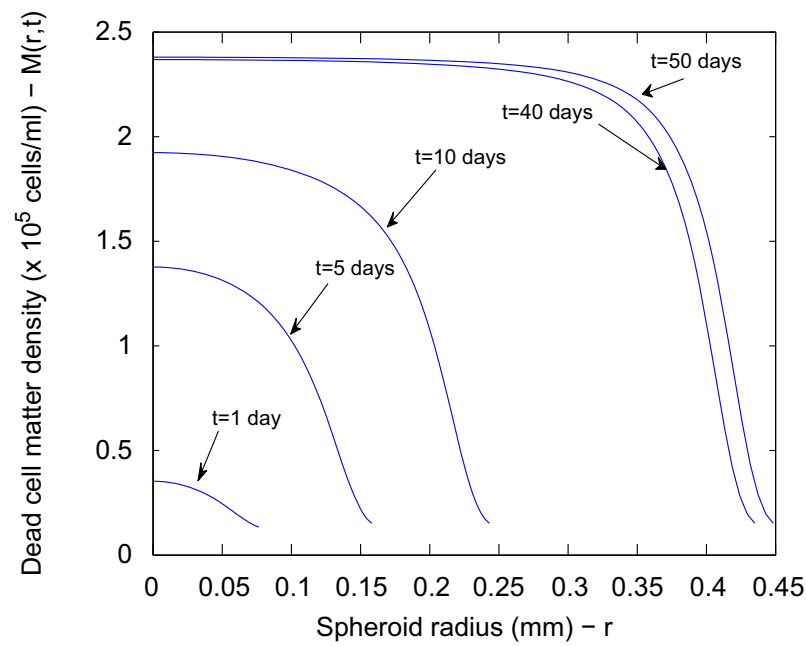

e

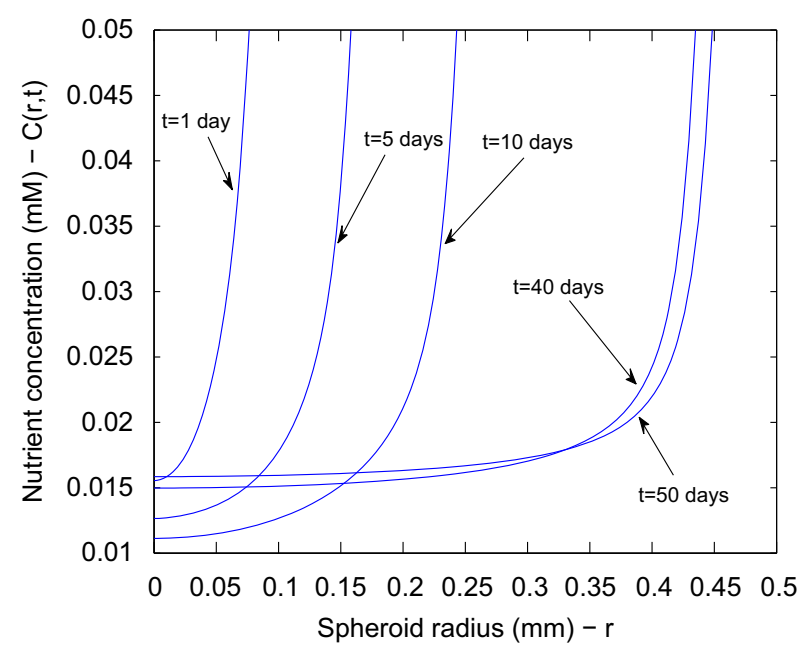

b

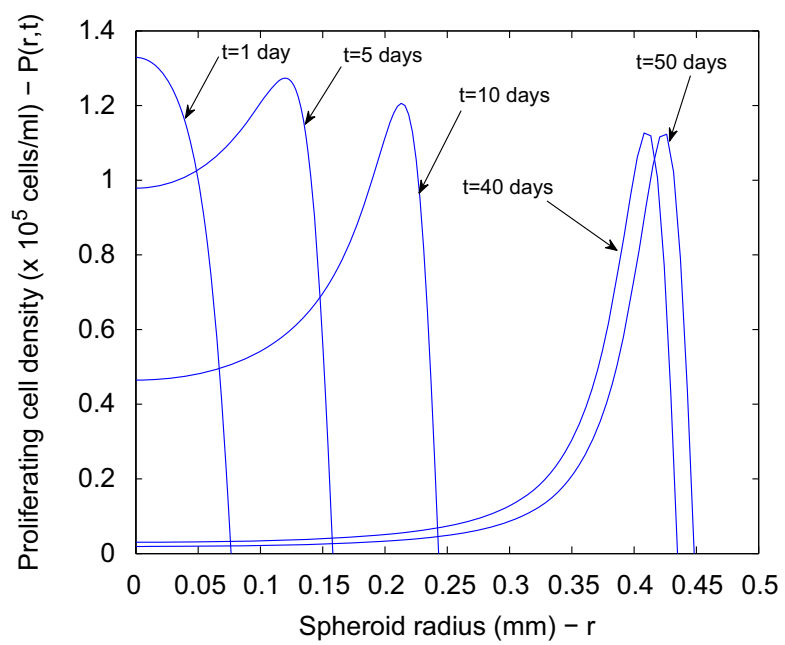

d

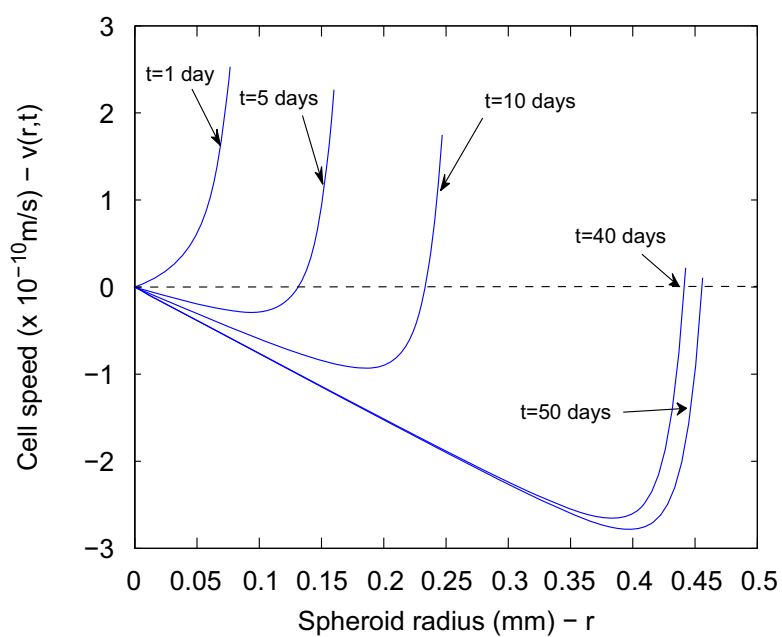

f

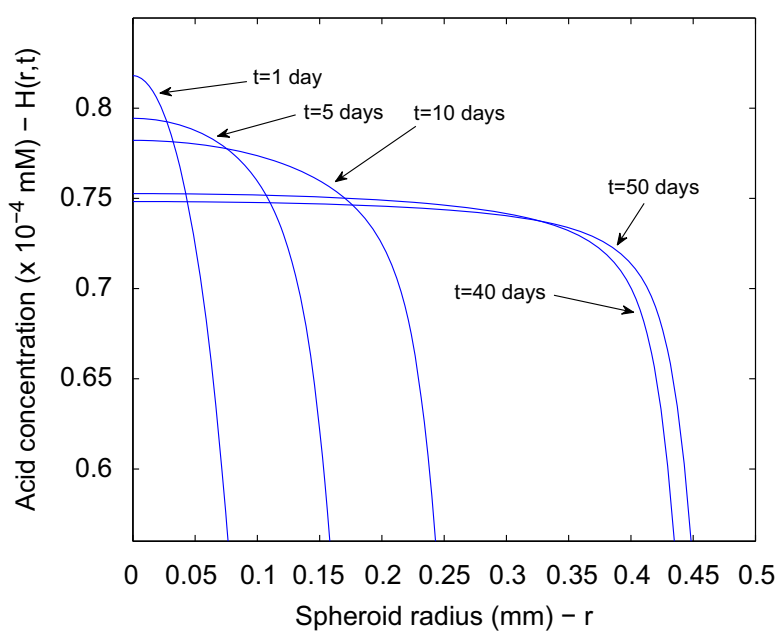

Fig. 1. Numerical simulations typical of the model showing the main dynamical behaviour during the first 50 days of growth (the tumour reaches a diameter of $0.896 \mathrm{~mm}$ ). The tumour obtains a final steady-state after a period of 80 days (with a $0.921 \mathrm{~mm}$ diameter) as seen in Fig. 2 . Parameter values used were as detailed in Table 2. (a) Proliferating cell distribution, $P(r, t)$. (b) Quiescent cell distribution, $Q(r, t)$. (c) Dead cell matter distribution, $M(r, t)$. (d) Cell velocity, $v(r, t)$. (e) Nutrient concentration, $C(r, t)$, and (f) Acid concentration, $H(r, t)$.

the difference in the rate of nutrient consumption by proliferating and quiescent cells. Fig. 3(a) and (b) compares the total radius and the radial velocity for three values of $\epsilon_{C}$ when hydrogen ions are present with $\epsilon_{H}=0.01$, and when no hydrogen ions are present. Increasing the value of $\epsilon_{C}$ results in a slower rate of growth and a smaller steady-state radius; an increased rate of oxygen 
a

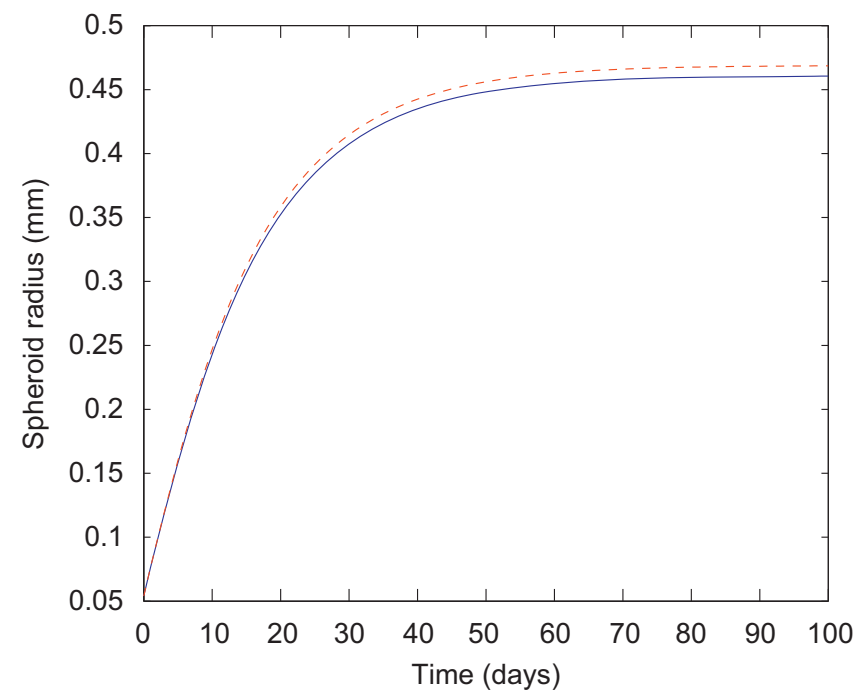

b

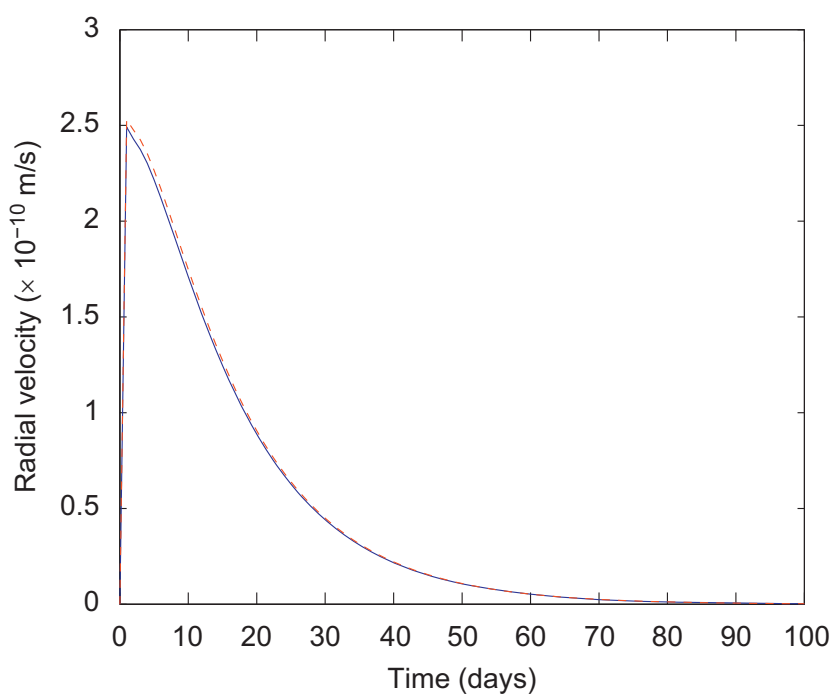

Fig. 2. Comparing the effect of acidosis on the size of the tumour in respect of the spheroid radius (a) and radial velocity (b), calculated from Eq. (23). The solid line denotes in the presence of acid and the dotted broken line is in the absence of acid ( $\left.k_{q}^{\prime}=0=k_{d}^{\prime}\right)$. In both cases all other parameters were held constant as defined in Table 2 . (a) Outer radius of the tumour $-\mathrm{R}(\mathrm{t})$. (b) Radial velocity of the tumour $-\mathrm{dR}(\mathrm{t}) / \mathrm{dt}$.

consumption by quiescent cells means the nutrient concentration falls more rapidly and more cells become quiescent. Thus there are fewer proliferating cells available to increase the tumour size.

However, increasing $\epsilon_{C}$ leads to a lower concentration of acid throughout the tumour as shown in Fig. 3(d), a rather counterintuitive result. We expect that decreasing the nutrient concentration would lead to a higher acid concentration via anaerobic metabolism (the term $\sigma_{h}(1-c)$ in Eq. (18)). Our model includes the well known biological result that a decrease in nutrient concentration leads to a higher rate of quiescence and therefore fewer proliferating cells. Since quiescent cells produce less acid than proliferating ones via $\epsilon_{H}$, our results indicate that this leads to a decrease in the acid concentration within the tumour. This result is demonstrated by setting $\epsilon_{H}=0$; as $\epsilon_{C}$ increases the acid concentration decreases (results not shown).

Our finding here shows the importance that the cell cycle state can have on the development of the tumour. By anaerobic metabolism alone, we would expect an increase in the acid concentration throughout the tumour. Including a description of proliferating and quiescent cells and the difference in acid production between them can counteract this effect. When the overall number of proliferating cells is greater than that of quiescent cells we expect a higher acid concentration as dictated by $\epsilon_{H} \ll 1$. When, however, the number of proliferating cells is less than the number of quiescent cells, less acid is produced and although the nutrient concentration is low, the effect of anaerobic metabolism on the acid concentration is secondary to that of the cell cycle state. We note that varying $\sigma_{h}$ alone had only a marginal affect on the acid concentration, i.e. $0 \leq \sigma_{h} \leq 1$ only resulted in a decrease in the spheroid radius of $R \sim 0.025$.

\subsubsection{Nutrient and acid effect on quiescence and necrosis}

The rates of cell quiescence and necrosis are dependent upon both the concentration of nutrient and acid. In this section we wish to quantify and compare the effect that nutrient and acid concentration have on the transition to these cell states.

In order to compare cell necrosis via a reduction in the nutrient or an increase in acid concentration, we define

$f_{d}=\frac{\int_{\Omega} k_{d}(1-c)-k_{d}^{\prime} h d \Omega}{\int_{\Omega} k_{d}(1-c)+k_{d}^{\prime} h d \Omega}$, where $\Omega$ is the volume of the tumour at steady-state. This relationship defines the ratio of the difference in cell death due to nutrient deprivation or acidosis with the total amount of cell death in the tumour due to each mechanism. When $f_{d}=1$ necrosis is dominated by a decrease in nutrient concentration, whilst when $f_{d}=-1$ acidosis has a greater effect than nutrient deprivation. We varied $k_{d}$ and $k_{d}^{\prime}$ according to $0.01 \leq k_{d} \leq 0.1$ and $10^{-4} \leq k_{d}^{\prime} \leq 10^{-3}$, respectively. Fig. 4(a) shows that necrosis is dominated by a decrease in the nutrient concentration rather than acidosis; a result of $k_{d}^{\prime}$ being three orders of magnitude less than $k_{d}$.

Cellular quiescence via either a decrease in nutrient concentration or increase in acid concentration, can be compared in a similar way such that

$f_{q}=\frac{\int_{\Omega} k_{q}(1-c)-k_{q}^{\prime} h d \Omega}{\int_{\Omega} k_{q}(1-c)+k_{q}^{\prime} h d \Omega}$

Similar to Eq. (28) this relationship defines the ratio of the difference in cell quiescence due to nutrient deprivation or acidosis with the total amount of cell quiescence in the tumour. Here we have varied $k_{q}$ and $k_{q}^{\prime}$ according to $0.1 \leq k_{q} \leq 1$ and $0.01 \leq k_{q}^{\prime} \leq 0.1$, respectively, as shown in Fig. 4(b). Similar to cell death via necrosis, cell quiescence is more sensitive to a change in acid concentration when the rate of quiescence due to the nutrient concentration is reduced.

\section{Summary and conclusions}

A mathematical model describing the growth of a MCTS and the effects that nutrient and acid concentrations have on the distribution of proliferating and quiescent cells and dead cell material (via necrosis and apoptosis) throughout the tumour has been formulated and solved.

We have found that the distribution of proliferating and quiescent cells can have important consequences on the amount of acid produced in the tumour. Decreased nutrient levels do not necessarily lead to excessive acid concentration via anaerobic metabolism. Instead, because quiescent cells produce less acid than proliferating cells, when the nutrient concentration is low, there are more quiescent and fewer proliferating cells and hence the increased effect of anaerobic metabolism is negligible. Our 
a

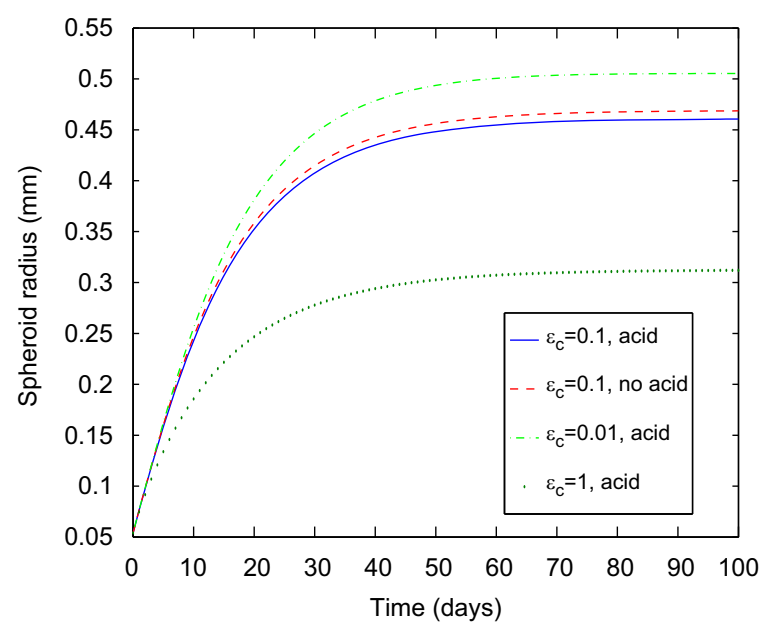

C

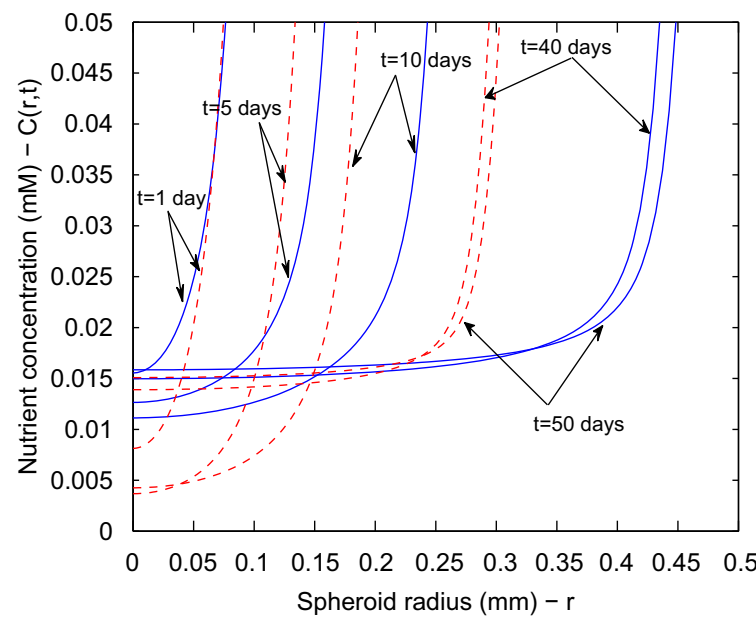

e

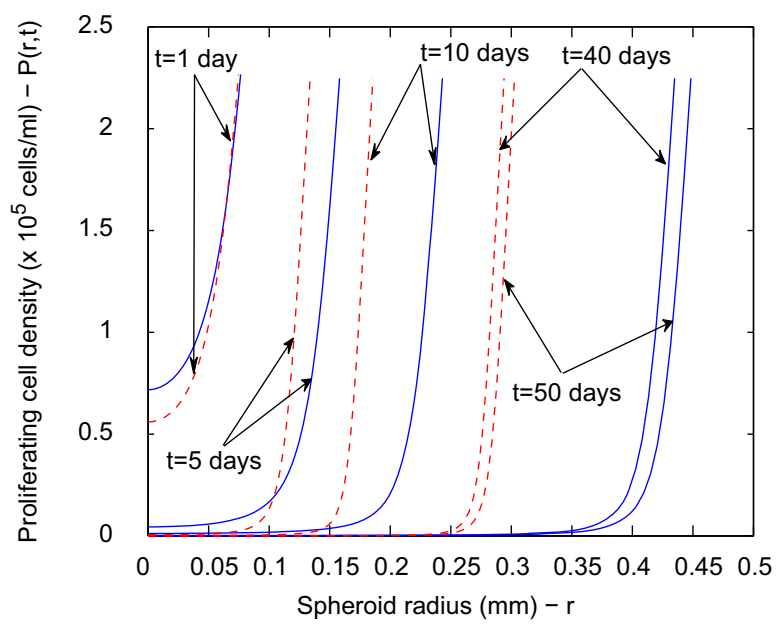

b

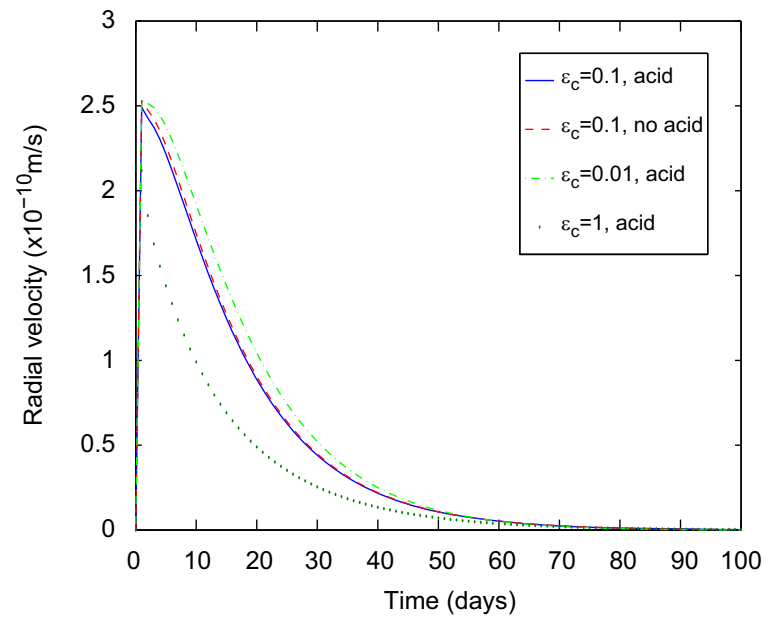

d

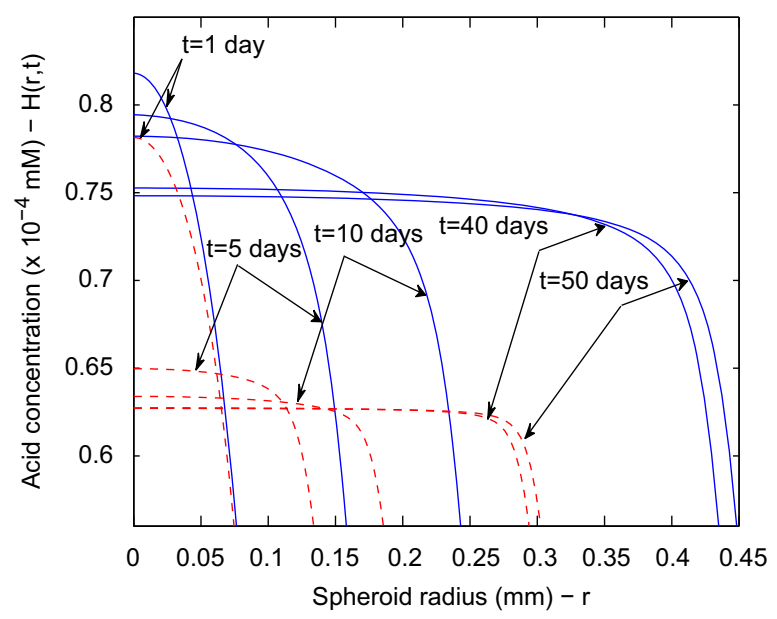

$f$

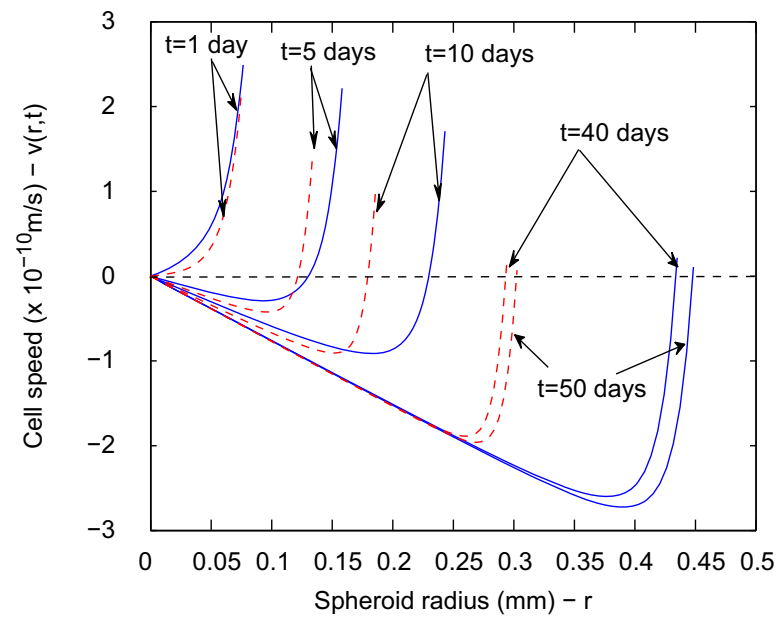

Fig. 3. (a) and (b) comparing the effect of different rates of nutrient consumption by quiescent versus proliferating cells on the overall size of the MCTS. (c)-(f) The effect the different rates have on nutrient and acid concentrations, proliferating cell density and velocity profiles. In (c)-(f) solid lines are for when $\epsilon_{c}=0.1$ and dotted lines when $\epsilon_{c}=1$. In both cases all other parameters were held constant as defined in Table 2. (a) Outer radius of the tumour $-R(t)$. (b) Radial velocity of the tumour - dR(t)/dt. (c) Nutrient concentration $-C(r, t)$. (d) Acid concentration $-H(r, t)$. (e) Proliferating cell distribution $-\mathrm{P}(\mathrm{r}, \mathrm{t})$. (f) Proliferating cell velocity - v(r, $\mathrm{t})$.

result here is dependent upon the rates of anaerobic metabolism and cells moving from the proliferating to quiescent cell compartments, which have been obtained from experimental data in the literature for a number of cancer cell lines.
Analysis of the model has also quantified the difference in cell quiescence and death due to either the local nutrient or acid concentration. In the case of necrosis we varied the rates of necrosis due to nutrient deprivation and acidosis, respectively, over one order 
a

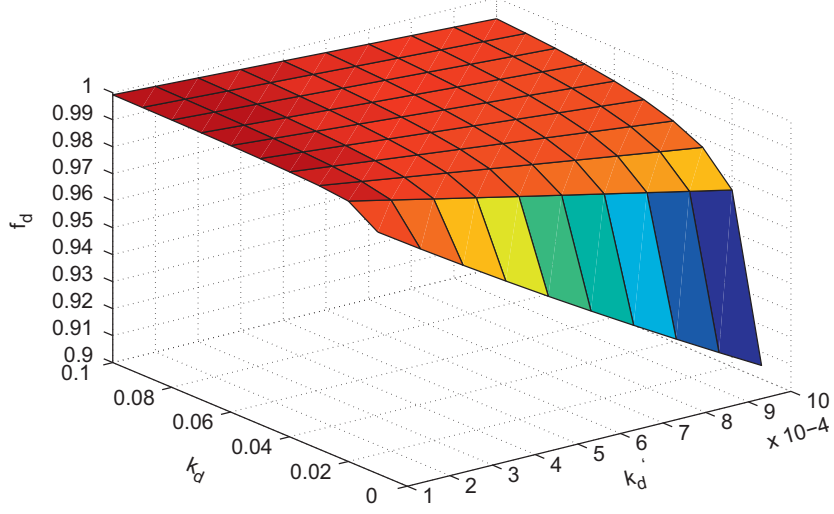

b

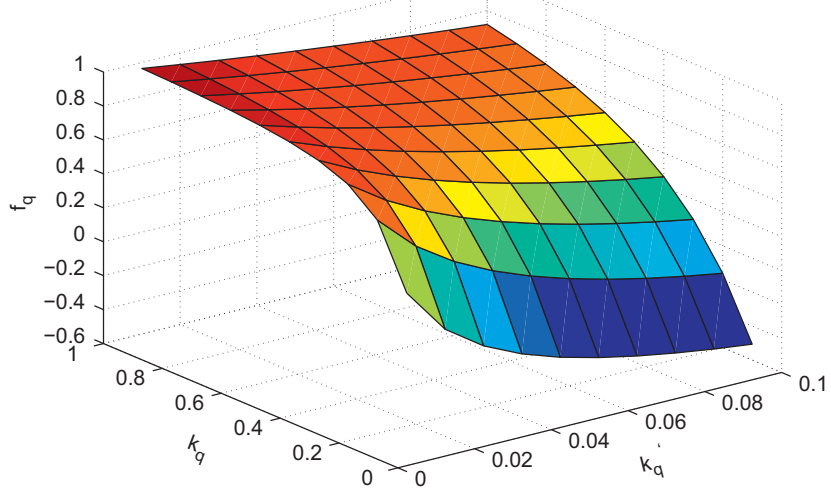

Fig. 4. The effect of nutrient and acid concentration on cell necrosis (a) and quiescence (b). Here $f_{d}$ quantitatively compares the effect of cell death via anaerobic metabolism versus acidosis to the total amount of cell death and is defined by Eq. (28) (when $f_{d}=1$ necrosis is dominated by a decrease in nutrient concentration, whilst when $f_{d}=-1$ acidosis dominates). Likewise Eq. (29) expresses a similar quantity in the context of cell quiescence. The results show that: (a) a decrease in nutrient concentration dominates over acidosis in terms of affecting cell death; and (b) quiescence via acidosis begins to dominate only after the rate of quiescence via anaerobic metabolism reaches a threshold level. Further details are provided in Section 4.1.1.

of magnitude. By then comparing the overall number of dead cells produced via each mechanism we were able to determine that both necrosis and quiescence are dominated by the nutrient rather than acid concentration. In the case of necrosis, it is clear that this is the result of considerable variation between the necrosis rates due to each mechanism. We note that altering our model to include proliferating cell death via necrosis and quiescent cell death via apoptosis does not alter the spatiotemporal distribution of cells in the tumour, but merely reduces the tumour size (results not shown).

Our results have shown that whilst nutrient and acid concentrations are independently important in affecting tumour growth, in understanding the 'bigger picture' one needs to account for the effect that the cell cycle state of the cells within the tumour has on their concentration. Effectively, the cell cycle state of the cells and respective nutrient and acid concentrations are thus interdependent systems. This result could be taken into account in respect of therapeutic strategies; it is important to quantify the ratio of proliferating and quiescent cells within the tumour in order to understand the effect of decreased nutrient concentrations. A reduction in nutrient concentration may not simply lead to an increase in the acid concentration within the tumour.

Furthermore, it has been shown that lowering acidity levels can inhibit cancer cell invasion (Robey et al., 2009) and therefore a greater understanding of the control of acidosis is required, especially if one envisions combination treatments involving angiogenesis, which will affect nutrient levels. One of the major challenges of transferring insights such as those gained from the present study into clinical practice will be to determine precisely where the patient is in parameter space as treatments may have very different effects on two different patients. Not only does this illustrate the difficulties in trying to develop general treatment protocols, but also the difficulties of drug testing without a profound knowledge of the underlying mechanisms affecting the spatiotemporal cell cycle state of cells within a tumour.

In the work presented here we have neither considered the effect of acidosis on the rate of apoptosis nor have we accounted for the difference in intracellular and extracellular acid and their subsequent effects. Such issues would need to be addressed in future work.

\section{Acknowledgements}

MJT is grateful to the Research Councils UK for providing him with an RCUK Fellowship during periods in which this research was undertaken. KS acknowledges the support of the BBSRC/ EPSRC grant BB/-C008219/1 "The Manchester Centre for Integrative Systems Biology (MCISB)". PKM was partially supported by a Royal Society-Wolfson Merit award and Award No. U54CA143970 from the National Cancer Institute. The content is solely the responsibility of the authors and does not necessarily reflect the views of the National Cancer Institute or the National Institutes of Health.

\section{References}

Anderson, A.R.A., 2005. A hybrid mathematical model of solid tumour invasion: the importance of cell adhesion. Math. Med. Biol. 22, 163-186.

Araujo, R.P., McElwain, D.L.S., 2004. A history of the study of solid tumour growth: the contribution of mathematical modelling. Bull. Math. Biol. 66 (5), 1039-1092.

Bajzer, Z., Vuk-Pavlovic, S., Huzak, M., 1997. Mathematical modeling of tumor growth kinetics. In: Adam, J.A. (Ed.), A Survey of Models for Tumor Immune System Dynamics. , Birkhaeuser, Boston, pp. 89-133.

Banaji, M., Tachtsidis, I., Delpy, D., Baigent, S., 2005. A physiological model of cerebral blood flow control. Math. Biosci. 194, 125-173.

Bertuzzi, A., Fasano, A., Gandolfi, A., Sinisgalli, C., 2007. ATP production and necrosis formation in a tumour spheroid model. Math. Model. Nat. Phenom. 2 (3), 30-46.

Bertuzzi, A., Gandolfi, A., Giovenco, M.A., 1981. Mathematical models of the cell cycle with a view to tumor studies. Math. Biosci. 53, 159-188.

Billy, F., Ribba, B., Saut, O., Morre-Trouilhet, H., Colin, T., Bresch, D., Boissel, J.P., Grenier, E., Flandrois, J.P., 2009. A pharmacologically based multiscale mathematical model of angiogenesis and its use in investigating the efficacy of a new cancer treatment strategy. J. Theor. Biol. 260, 545-562.

Bredel-Geissler, A., Karbach, I., Walenta, S., Vollrath, L., Mueller-Klieser, W., 1992 Proliferation-associated oxygen consumption and morphology of tumor cells in monolayer and spheroid culture. J. Cell. Physiol. 153, 44-52.

Breward, C.J.W., Byrne, H.M., Lewis, C.E., 2002. The role of cell-cell interactions in two-phase model for avascular tumour growth. J. Math. Biol. 45, 125-152.

Burton, A.C., 1996. Rate of growth of solid tumors as a problem of diffusion. Growth 30, 157-176.

Casciari, J.J., Sotirchos, S.V., Sutherland, R.M., 1992. Variations in tumor cell growth rates and metabolism with oxygen concentration, glucose concentration, and extracellular pH. J. Cell. Physiol. 151, 386-394.

Cojocaru, L., Agur, Z., 1992. A theoretical analysis of interval drug dosing for cellcycle-phase-specific drugs. Math. Biosci. 109 (1), 85-97.

Forbes, N.S., Venkatasubramanian, R., Henson, M.A., 2006. Incorporating energy metabolism into a growth model of multicellular tumor spheroids. J. Theor. Biol. 242 (2), 440-453.

Gatenby, R.A., Smallbone, K., Maini, P.K., Rose, F., Averill, J., Nagle, R.B., Worrall, L., Gillies, R.J., 2007. Cellular adaptations to hypoxia and acidosis during somatic evolution of breast cancer. Br. J. Cancer 97, 646-653.

Gillies, R.J., Raghunand, N., Garcia-Martin, M.L., Gatenby, R.A., 2004. pH imaging. IEEE Eng. Med. Biol. 23, 57-64.

Gillies, R.J., Raghunand, N., Karczmar, G.S., Bhujwalla, Z.M., 2002. MRI of the tumor microenvironment. J. Magn. Reson. Imag. 16, 430-450.

Greenspan, H.P., 1972. Models for the growth of a solid tumor by diffusion. Stud. Appl. Math. 51 (4), 317-340. 
Hillen, T., De Vries, G., Jong, J.F., Finlay, C., 2010. From cell population models to tumor control probability: Including cell cycle effects. Acta Oncol. 49, 1315-1323.

Höckel, M., Vaupel, P., 2001. Tumor hypoxia: Definitions and current clinical, biologic, and molecular aspects. J. Natl. Cancer Inst. 93, 266-276.

Jeon, J., Quaranta, V., Cummings, P.T., 2010. An off-lattice hybrid discretecontinuum model of tumor growth and invasion. Biophys. J. 98, 37-47.

Kumei, Y., Nakajima, T., Sato, A., Kamata, N., Enomoto, S., 1989. Reduction of $\mathrm{G}_{1}$ phase duration and enhancement of $c$-myc gene expression in HeLa cells at hypergravity. J. Cell Sci. 93, 221-226.

Kunz-Schugart, L.A., Kreutz, M., Knuechel, R., 1998. Multicellular spheroids: A three-dimensional in vitro culture system to study tumour biology. Int. J. Exp. Pathol. 79, 1-23.

Mahmood, M.S., Mahmood, S., Dobrota, D., 2011. Formulation and numerical simulations of a continuum model of avascular tumor growth. Math. Biosci. $231,159-171$

Meikrantz, W., Schlegel, R., 1995. Apoptosis and the cell cycle. J. Cell. Biochem. 58, 160-174.

Mueller-Klieser, W.F., 1997. Three-dimensional cell cultures: from molecular mechanisms to clinical applications. Am. J. Physiol. 273, 1109-1123 4(1).

Netti, P.A., Baxter, L.T., Boucher, Y., Skalak, R., Jain, R.K., 1995. Time-dependent behaviour of interstitial fluid pressure in solid tumours: implications for drug delivery. Cancer Res. 55, 5451-5458.

Nichols, M.G., Foster, T.H., 1994. Oxygen diffusion and reaction kinetics in the photodynamic therapy of multicell tumour spheroids. Phys. Med. Biol. 39, 2161-2181.

Park, H.J., Lyons, J.C., Ohtsubo, T., Song, C.W., 1999. Acidic environment causes apoptosis by increasing caspase activity. Br. J. Cancer 80, 1892-1897.

Patel, A.A., Gawlinski, E.T., Lemieux, S.K., Gatenby, R.A., 2001. A cellular automaton model of early tumor growth and invasion. J. Theor. Biol. 213, 315-331.

Racker, E., 1974. History of the Pasteur effect and its pathobiology. Mol. Cell. Biochem. 5, 17-23.
Robey, I.F., Baggett, B.K., Kirkpatrick, N.D., Roe, D.J., Sloane, B.F., Dosescu, J., Hashim, A.I., Morse, D.L., Raghunand, N., Gatenby, R.A., Gillies, R.J., 2009. Bicarbonate increases tumor $\mathrm{pH}$ and inhibits spontaneous metastases, cancer research. Cancer Res. 69, 2260-2268.

Roose, T., Chapman, S.J., Maini, P.K., 2007. Mathematical models of avascular tumour growth: A review. SIAM Rev. 49, 179-208.

Schornack, P.A., Gillies, R.J., 2003. Contributions of cell metabolism and $\mathrm{H}^{+}$diffusion to the acidic $\mathrm{pH}$ of tumors. Neoplasia $5,135-145$.

Smallbone, K., Gatenby, R.A., Gillies, R.J., Maini, P.K., Gavaghan, D.J., 2007. Metabolic changes during carcinogenesis: potential impact on invasiveness. J. Theor. Biol. 244, 703-713.

Tanaka, M.L., Debinski, W., Puri, I.K., 2009. Hybrid mathematical model of glioma progression. Cell. Prolif. 42 (5), 637-646.

Tindall, M.J., Please, C.P., 2007. Modelling the cell cycle and cell movement in multicellular tumours spheroids. Bull. Math. Biol. 69 (4), 1147-1165.

Tindall, M.J., Please, C.P., Peddie, M.J., 2008. The formation of necrotic regions in avascular tumours. Math. Biosci. 211 (1), 34-55.

Tung, Y., Hsiao, A.Y., Allen, S.G., Torisawa, Y., 2011. High-throughput 3D spheroid culture and drug testing using a 384 hanging drop array. Analyst 136, 473-478.

Warburg, O., 1930. The Metabolism of Tumours. Constable Press, London.

Ward, J.P., King, J.R., 1997. Mathematical modelling of avascular tumour growth. IMA J. Math. Appl. Med. Biol. 14, 36-69.

Ward, J.P., King, J.R., 1998. Mathematical modelling of avascular-tumour growth ii: modelling growth saturation. IMA J. Math. Appl. Med. Biol. 15, 1-42.

Williams, A.C., Collard, T.J., Paraskeva, C., 1999. An acidic environment leads to p53 dependent induction of apoptosis in human adenoma and carcinoma cell lines: implications for clonal selection during colorectal carcinogenesis. Oncogene 18, 3199-3204.

Zhao, Y.C., Ricci, P.F., 2010. Modeling dose-response at low dose: a systems biology approach for ionization radiation. Dose-Response 8, 456-477. 